\title{
Ultrasound Strain Imaging to Assess the Biceps Brachii Muscle in Chronic Poststroke Spasticity
}

Jing Gao, MD (D), Johnson Chen, MD, Michael O'Dell, MD, Pai-Chi Li, PhD, Wen He, MD,
Li-Juan Du, MD, Jonathan M. Rubin, MD, PhD D Dilliam Weitzel, MD, Robert Min, MD

Received September 20, 2017, from the Departments of Radiology (J.G., J.C., R.M.) and Rehabilitation Medicine (M.O.), Weill Cornell Medicine, New York, New York USA; Rocky Vista University, Ivins, Utah USA (J.G.); Electrical Engineering College, National Taiwan University, Taipei, Taiwan (P.-C.L.); Beijing Tiantan Hospital, Capital Medical University, Beijing, China (W.H., L.J.D.); Department of Radiology, University of Michigan, Ann Arbor, Michigan USA (J.M.R.); and Veterans Affairs Ann Arbor Health System, Ann Arbor, Michigan USA (W.W.). Manuscript accepted for publication November 15, 2017.

We thank Siemens Medical Solutions for lending the ultrasound scanner to support the study.

Address correspondence to Jing Gao, $M D$, Ultrasound in Education \& Research, Rocky Vista University, 255 E Center St, Room C286, Ivins, UT 84738 USA.

E-mail: jgao@rvu.edu

Abbreviations

US, ultrasound

doi:10.1002/jum.14558
Objectives - The aim of the study was to assess the feasibility of ultrasound strain imaging in characterizing the biceps brachii muscle in chronic poststroke spasticity.

Methods-We prospectively analyzed strain imaging data from bilateral biceps brachii muscles in 8 healthy volunteers and 7 patients with poststroke chronic spasticity. Axial deformations of the biceps brachii muscle and overlying subcutaneous tissue were produced by external compression using a sandbag $(1.0 \mathrm{~kg})$ attached to a transducer. The lengthening and shortening of the biceps brachii muscle and subcutaneous tissue were produced by manual passive elbow extension (from $90^{\circ}$ to $0^{\circ}$ ) and flexion (from $0^{\circ}$ to $90^{\circ}$ ), respectively. We used offline 2-dimensional speckle tracking to estimate axial and longitudinal strain ratios (biceps brachii strain/subcutaneous tissue strain), and the longitudinal tissue velocity of the biceps brachii muscle. Statistical analyses included analysis of variance for testing differences in strain imaging parameters among healthy, nonspastic, and spastic biceps brachii muscles, the Bonferroni correction for further testing differences in US strain imaging among paired groups (healthy versus spastic, nonspastic versus spastic, and healthy versus nonspastic), and the Pearson correlation coefficient for assessing the intraobserver reliability of performing strain imaging in stroke survivors.

Results - The differences in strain imaging parameters between healthy and spastic and between nonspastic and spastic biceps brachii muscles were significant at both $90^{\circ}$ elbow flexion and maximal elbow extension $(P<.01)$. There was no significant difference in axial strain ratios at $90^{\circ}$ of elbow flexion or longitudinal tissue velocities between healthy and nonspastic muscles $(P>.05)$. The intraobserver reliability of performing strain imaging in stroke survivors was good $(r=0.85 ; P<.01)$.

Conclusions-Ultrasound strain imaging seems to be feasible for characterizing the biceps brachii muscle in chronic poststroke spasticity.

Key Words - biceps brachii muscle; elastography; musculoskeletal (diagnostic); poststroke spasticity; ultrasound strain

A pproximately two-thirds of 15 million stroke survivors require rehabilitation for the consequences of poststroke spasticity each year worldwide. ${ }^{1}$ Spasticity primarily affects the muscles surrounding the elbow (79\%), wrist (66\%), and ankle (66\%). ${ }^{2,3}$ Accurately characterizing and quantifying the mechanical behavior of spastic muscle may help us better understand the relationship between the clinical manifestation and underlying pathophysiologic mechanism leading to spastic muscle. Furthermore, an effective measure of spasticity is needed to determine the necessity for and efficacy of potential interventions in clinical management. ${ }^{4,5}$ To date, this 
process remains challenging because of the lack of a reference standard. ${ }^{6,7}$ The commonly used modified Ashworth Scale and Tardieu Scale in clinical assessment of muscle spasticity are subjective and nonquantitative. ${ }^{8}$ Electromyography measures electrical activity of muscles without imaging guidance. ${ }^{9}$ In individuals with poststroke spasticity, the stiffness increases and motion dynamics decrease in spastic muscle. Poststroke muscle spasticity and its negative secondary effects (eg, contractures, limited joint range of motion, and pain) limit musculoskeletal function, which affects daily living activities. ${ }^{1,10,11}$ It would be ideal to have a noninvasive imaging technique to quantify the mechanical properties and dynamic movement of spastic muscle to assist clinicians in the diagnosis of spasticity, monitoring of disease progression, and evaluation of the treatment response in stroke rehabilitation. ${ }^{5}$

Ultrasound (US) strain imaging has proven to be a useful technique for the assessment of mechanical properties (stiffness) of skeletal muscle. ${ }^{12-14}$ With 2dimensional speckle tracking, the axial strain estimates tissue deformation along the US beam, which is the change in axial tissue length relative to its original length in the direction of the compression. ${ }^{15-17}$ The longitudinal strain is the local muscle deformation and displacement parallel to the longitudinal direction of muscle fascicles, resulting from muscle fiber lengthening or shortening produced by passive limb joint movement. ${ }^{18}$ The strain is high in "softer" or more-elastic tissue, and it is low in "stiffer" or less-elastic tissue. However, little is known about the strain imaging technique in assessing skeletal muscle dynamic motion. ${ }^{18}$ We have reported the feasibility of strain imaging in determining rigid biceps brachii muscle stiffness in Parkinson disease. ${ }^{19}$ We have also observed high interobserver and intraobserver agreement of strain imaging in assessing passive biceps brachii dynamic motion in healthy adults. ${ }^{20}$ The aim of this study was to assess the feasibility of strain imaging for assessing spasticity in biceps brachii muscles of stroke survivors.

\section{Materials and Methods}

The Internal Review Board at Weill Cornell Medicine approved this study (No. 1601016917), and all participants provided written informed consent.

Ultrasound strain imaging of bilateral biceps brachii muscles was performed in 8 healthy volunteers and 8 individuals with poststroke spasticity of the upper limbs.
The study inclusion criteria for healthy controls included age of 40 to 60 years (an age group with high relevance with regard to vascular events) with normal physical examination findings, no history of trauma or surgery of the arm, no neuromuscular disorders, and no medication that may have affected muscle stiffness and movement. The study inclusion criteria for patients with chronic poststroke spasticity included age older than 18 years, time from the vascular event to the US examination of greater than 6 months, ${ }^{4,7}$ no history of arm trauma or surgery, ability to sign written informed consent and tolerate passive elbow movement in the US examination, modified Ashworth Scale score, and Tardieu Scale score. All patients received care from a physician in the Department of Rehabilitation Medicine of Weill Cornell Medicine as the standard of care for the treatment of their poststroke spasticity.

An Acuson S3000 HELX US system equipped with a 9L4 linear array transducer (Siemens Medical Solutions, Mountain View, CA) was used to acquire grayscale images and real-time US data of biceps brachii deformation and movements. The participant was placed in the supine position, and the arm was relaxed with the forearm supinated. To capture real-time US data of biceps brachii axial deformation and longitudinal displacement, transmission gel was applied, and the US transducer was in the long axis placed on the skin, which was elongated with an underlying longitudinal section of the biceps brachii (biceps belly, middle part to distal part of the muscle) fiber. ${ }^{20}$ We began scanning with grayscale imaging to observe morphologic characteristics along a longitudinal section of the biceps brachii muscle. We then acquired real-time US data, including bilateral biceps brachii axial deformation and longitudinal displacement sequences.

\section{Real-time US Data Acquisition}

Machine settings for acquiring real-time US data were standardized to capture grayscale imaging cine loops (5 seconds) of axial deformation and longitudinal displacement of the biceps brachii muscle. The standardized machine settings for acquiring US data included a maximum image depth of $4 \mathrm{~cm}$, scanning frequency of 7 $\mathrm{MHz}$, single image focus, tissue harmonic imaging, time/space of 0 , and speckle reduction function turned off to reach real-time frame rates of more than 40 frames per second and to enhance contrast resolution of grayscale images. ${ }^{20}$ 
Biceps brachii axial deformation parallel to the emission sound beam was produced by external compression on the skin and underlying longitudinal section of the biceps brachii muscle (Figure 1A) using a 1.0-kg sandbag tied onto the transducer as the compression force. ${ }^{20}$ The operator held the transducer steady on the underlying skin to avoid out-of-plane motion and any additional force by the operator. A cine loop capturing real-time US data of biceps brachii deformation and relaxation at an elbow angle of $90^{\circ}$ (using a $90^{\circ}$ angled rest) and at the maximal elbow extension (full extension at a $0^{\circ}$ elbow angle in healthy controls and possible maximum in stroke survivors) was recorded twice in each muscle.
Biceps brachii displacement perpendicular to the emission sound beam was imaged with a free hand (without sandbag) holding the transducer normal to the skin and underlying biceps brachii muscle while an observer manually positioned the elbow in flexion or extension. Longitudinal strain measurements, displacements, and velocities were measured parallel to the muscle fascicles, which are seen as echogenic bands on the US images in the longitudinal orientation (Figure 1B). Cine loops capturing 5-second real-time US data of passive biceps brachii movement in flexion (elbow angle from $0^{\circ}$ to $90^{\circ}$ ) and extension (elbow extension from $90^{\circ}$ to $0^{\circ}$ or extending maximally without causing pain)

Figure 1. Real-time grayscale images of a longitudinal section of the biceps brachii muscle and subcutaneous tissue were captured for offline strain estimation. With 2-dimensional speckle tracking, the regions of interest for estimating axial and longitudinal biceps brachii and reference strain were selected. Biceps brachii axial strain (A) represents deformations along the direction of emission sound beam in a 20-mm anteroposterior region (cyan, red, and yellow dotted lines) of the muscle, and reference axial strain represents the deformation in 5-mm anteroposterior subcutaneous soft tissue (purple dotted line) under external compression. Longitudinal strain (B) estimates the tissue displacement moving perpendicular to the direction of emission the sound beam and elongation with muscle fiber and fascicles (yellow arrow) after manual elbow extension (from $90^{\circ}$ to $0^{\circ}$ ) and flexion (from $0^{\circ}$ to $90^{\circ}$ ). Biceps brachii and reference longitudinal strains represent longitudinal displacements in $10 \mathrm{~mm}$ of muscle (red dotted line) and $10 \mathrm{~mm}$ of subcutaneous tissue (cyan dotted line), respectively. The data quality index (C) was used to assess the validity of acquired real-time US data for estimating tissue strain using correlation coefficient method in speckle tracking. The data quality index of greater than 0.95 in this graph indicates that the real-time US data are valid for strain estimation.
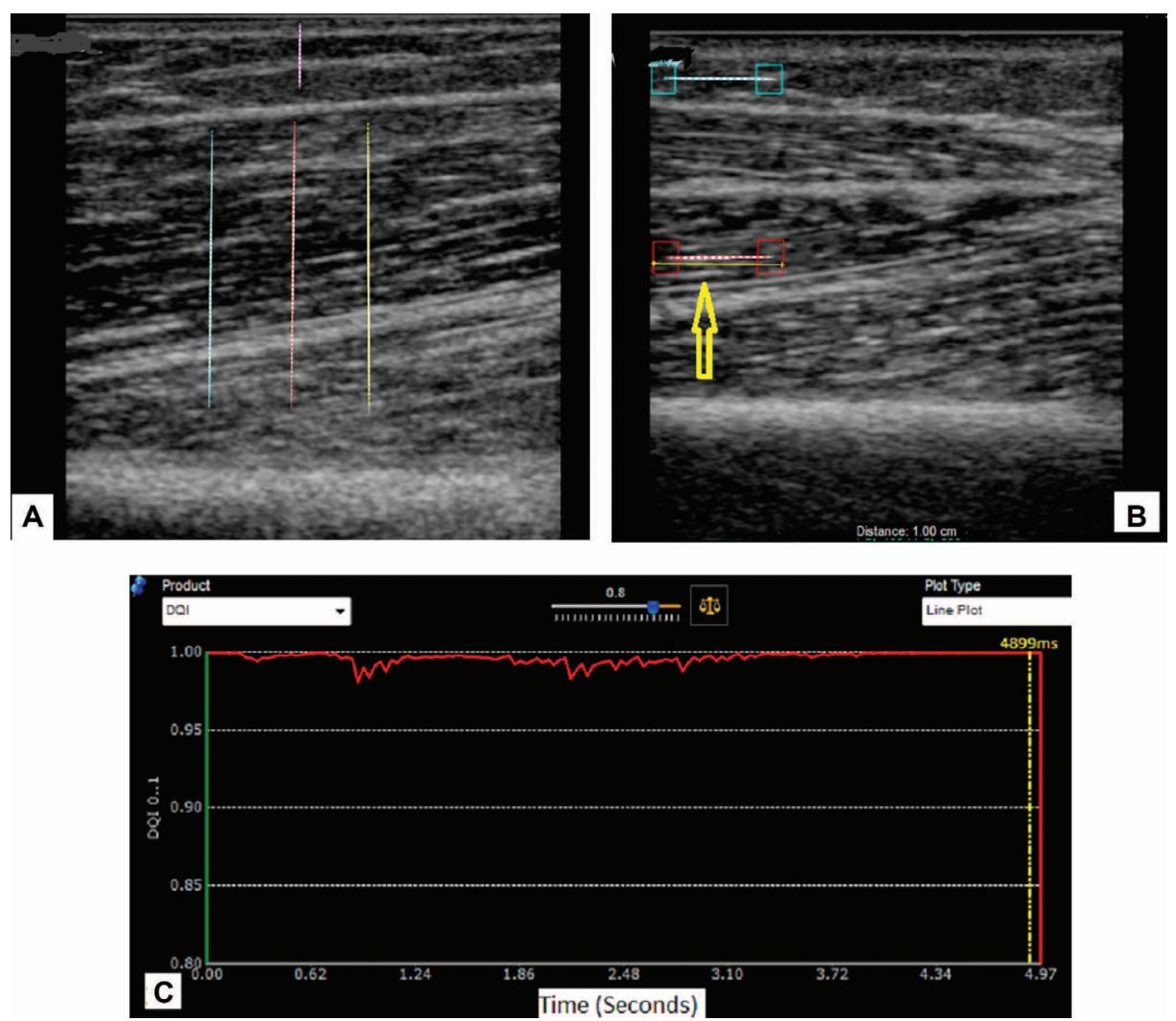
were recorded twice in each muscle. During real-time US data capturing, special attention was paid to avoid any out-of-plane motion by both the investigator and participant and to maintain consistent elbow motion to minimize variation in performing manual elbow flexion and extension. All real-time grayscale image cine loops in the Digital Imaging and Communications in Medicine format were exported via a universal serial bus and then transferred to a personal computer for offline processing.

\section{Two-Dimensional Speckle Tracking to Estimate Biceps Brachii Strain and Tissue Velocity}

Strain imaging parameters (axial strain, longitudinal strain, and tissue velocity) of the biceps brachii muscle (Figure 1, A and B) were estimated by 2-dimensional speckle-tracking software (EchoInsight; Epsilon Imaging, Ann Arbor, MI). With the use of phase-sensitive cross-correlation methods for speckle tracking, ${ }^{17,21}$ the data quality index $(0-1)$ is the measure of the frame-toframe correlation as an indication of the accuracy of motion tracking between frames in axial and lateral directions. ${ }^{22}$ In this study, a data quality index of greater than 0.9 (Figure 1C) was considered valid real-time US data for strain estimation. ${ }^{16,23}$ We measured biceps brachii strain by the formula $\left(L_{1}-L_{0}\right) / L_{0}$, where $L_{0}$ was the initial distance, and $L_{1}$ was the altered distance of the biceps brachii muscle while measured. ${ }^{15-17}$

Axial biceps brachii and reference strains represent the anteroposterior deformation in $20 \mathrm{~mm}$ of the biceps brachii muscle and $5 \mathrm{~mm}$ of subcutaneous tissue, respectively. Longitudinal biceps brachii and reference strains directly represent 1-dimensional tissue lengthening or shortening in $10 \mathrm{~mm}$ of the biceps brachii muscle and $10 \mathrm{~mm}$ of subcutaneous tissue. The relationship between the strain value (y-axis) and its corresponding time ( $\mathrm{x}$-axis) is displayed as time-strain curves on strain graphs (Figures 2 and 3). A positive strain value $(>0)$ represents muscle lengthening (elbow extension from $90^{\circ}$ to $0^{\circ}$; Figure $3, \mathrm{~A}$ and B). A negative strain value $(<0)$ represents muscle shortening (elbow flexion from $0^{\circ}$ to $90^{\circ}$; Figure 3, C and D). In this study, the strain ratio (axial and longitudinal) was the relationship of the maximum strain in the biceps brachii muscle (axial or longitudinal) with the maximum strain in the subcutaneous tissue.

In addition, by using the correlation coefficient method to track the speckle pattern between matching kernels in consecutive real-time B-mode frames, 1-dimensional propagation of mechanical waves and transient tissue velocities during muscle movement can be quantified. ${ }^{24,25}$ Longitudinal biceps brachii tissue velocity represents a rapid mechanical lengthening or shortening in longitudinal biceps brachii concentric or eccentric movement. The peak velocity may be considered the most valid measure for estimating muscle dynamics because the tissue velocity is a temporal derivative of tissue displacement. A time-velocity curve (Figure 4) displays the magnitude

Figure 2. Time-strain graphs show the relationship of the axial biceps brachii strain to axial subcutaneous soft tissue (reference) strain estimated by 2-dimensional speckle-tracking software. The axial strain ratio is defined as the average of 3 biceps brachii strains (cyan, red, and yellow dotted lines) divided by the reference strain (purple line). There is an apparent significant difference in the axial strain ratio between the biceps brachii muscles in a 56-year-old healthy volunteer (A) and a 42-year-old stroke survivor (B) with a modified Ashworth Scale score of 2 and a Tardieu Scale score of 2 (axial strain ratio, 5.2 versus 3.1). The difference in reference strain between the participants is not significant. The result suggests that the spastic biceps brachii muscle is stiffer and less elastic than the healthy muscle.
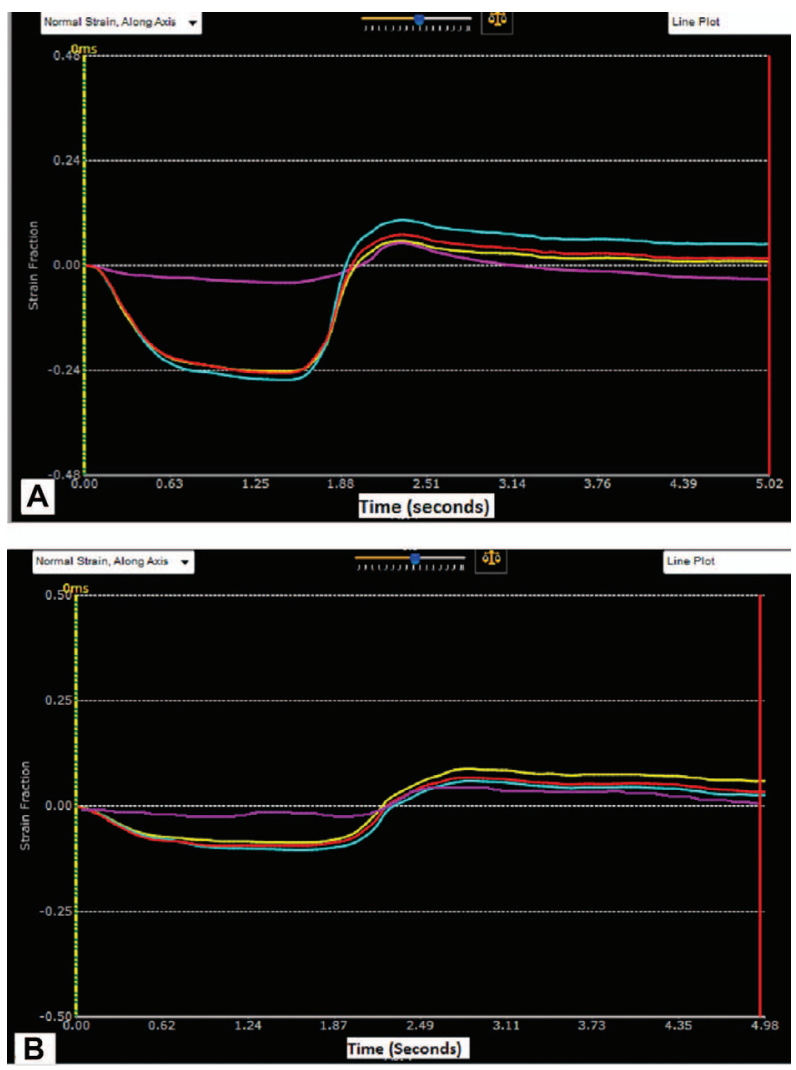
of the longitudinal muscle displacement over time based on the initiation. ${ }^{25,26}$

A single investigator (J.G.) successfully performed strain imaging twice for each biceps brachii muscle in all participants. The interval between the US data acquisitions was 2 minutes. She was blinded to results of modified Ashworth Scale and Tardieu Scale examinations.

\section{Modified Ashworth Scale and Tardieu Scale}

Modified Ashworth Scale and Tardieu Scale examinations were performed on all stroke survivors immediately after US examinations by an occupational therapist with 10 years of experience in using both scales and administering therapy for poststroke spasticity. The occupational therapist was blinded to strain imaging results.
The modified Ashworth Scale for the arm in a quick stretch is scored as follows ${ }^{27}: 0$, no increase in muscle tone; 1 , slight increase in muscle tone, manifested by a catch and release or by minimal resistance at the end of the range of motion when the affected part is moved in flexion or extension; $1+$, slight increase in muscle tone, manifested by a catch, followed by minimal resistance throughout the remainder (less than half) of the region of movement; 2 , more marked increase in muscle tone through most of the region of movement but easily moved; 3 , considerable increase in muscle tone, with difficult passive movement; and 4, affected part rigid in flexion or extension.

The Tardieu Scale incorporates a passive range of motion and a quick stretch that is graded as follows ${ }^{28}: 0$,

Figure 3. Time-strain graphs show longitudinal strains of the biceps brachii muscle and reference subcutaneous tissue during manual elbow movement and estimated by 2-dimensional speckle-tracking software (Figure 1b). A positive strain value ( $>0$ ) represents tissue lengthening during extension of the elbow angle from $90^{\circ}$ to $0^{\circ}(\mathbf{A}$ and $\mathbf{B})$. A negative strain value $(<0)$ represents tissue shortening during flexion of the elbow angle from $0^{\circ}$ to $90^{\circ}(\mathbf{C}$ and $\mathbf{D}$ ). The longitudinal strain ratio is defined as the biceps brachii strain divided by the reference strain. The longitudinal strain ratio is significantly higher in a healthy biceps brachii muscle ( $\mathbf{A}$ and $\mathbf{C}$ ) than in a spastic muscle with a modified Ashworth Scale score of $1+$ and a Tardieu Scale score of 2 (B and D) during elbow extension (A versus B) and during elbow flexion (C versus D).
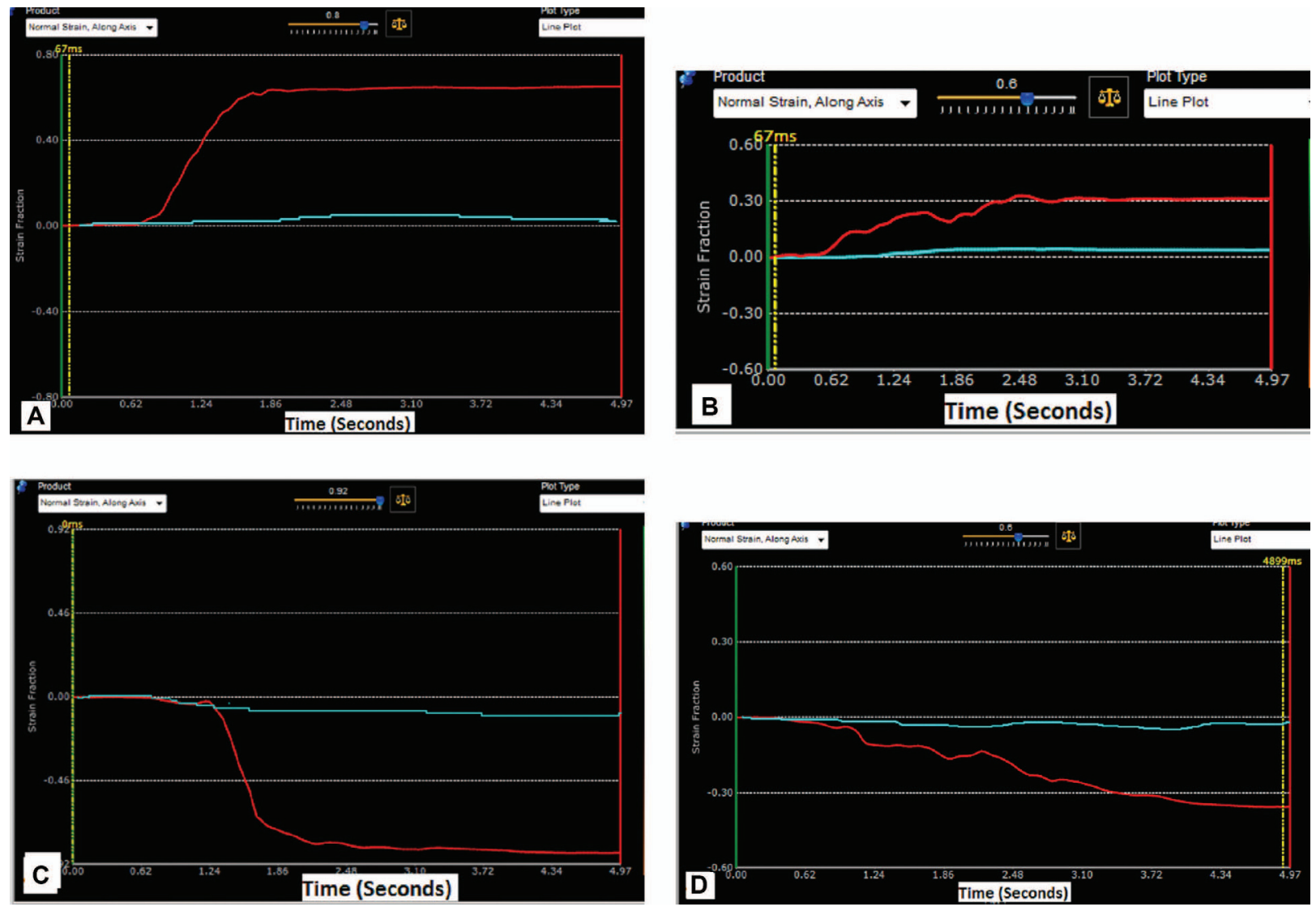
no resistance throughout passive movement; 1 , slight resistance throughout passive movement; 2 , clear catch at a precise angle, interrupting passive movement, followed by release; 3 , fatigable clonus (a series of involuntary rhythmic muscular contractions and relaxations; $<10$ seconds when maintaining pressure) occurring at a precise angle, followed by release; and 4, sustained clonus ( $>10$ seconds when maintaining pressure) occurring at a precise angle.

\section{Statistical Analyses}

All variables, including biceps brachii axial strain, longitudinal strain, tissue velocity, and age of the healthy controls and stroke survivors were characterized as mean \pm standard deviation. One-way analysis of variance was used to test the differences in strain imaging parameters among healthy, nonspastic, and spastic biceps brachii muscles. The Bonferroni correction was then applied to test the differences among paired groups (healthy versus spastic, nonspastic versus spastic, and healthy versus nonspastic).

Box-and-whisker plots were used to display the differences in strain imaging among the healthy, nonspastic,

Figure 4. Time-velocity graphs show biceps brachii (red line) and subcutaneous (cyan line) tissue velocity during tissue lengthening (elbow extension from $90^{\circ}$ to $0^{\circ}$ ). The notion is that the peak biceps brachii tissue velocity is significantly higher in the healthy muscle (A) than in the spastic muscle (B; 2.9 versus $1.7 \mathrm{~cm} / \mathrm{s}$ ). It should be noted that the velocity scale in $\mathbf{A}(2.5 \mathrm{~cm} / \mathrm{s})$ is different from that in $\mathbf{B}$ $(1.0 \mathrm{~cm} / \mathrm{s})$.
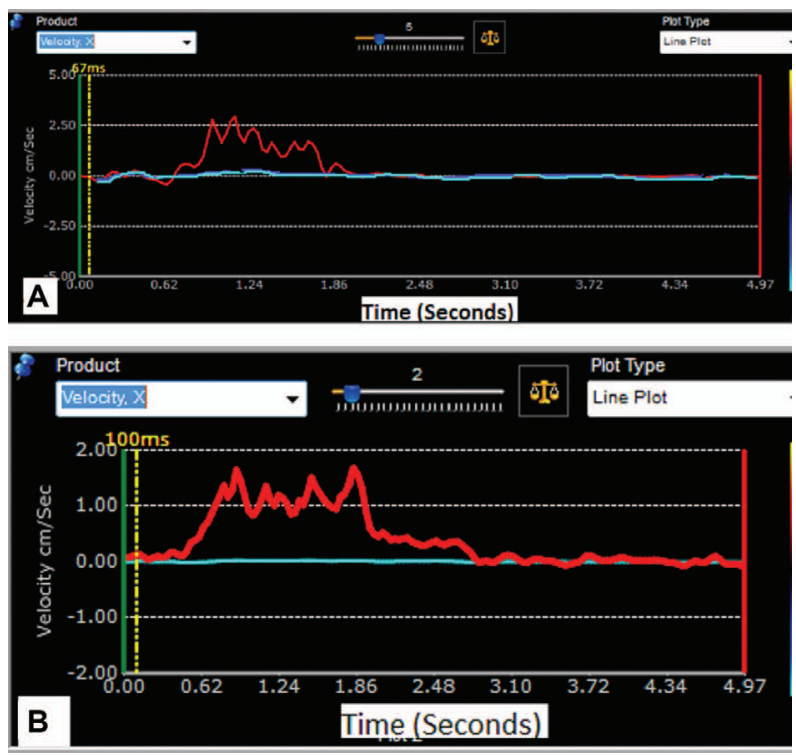

and spastic biceps brachii muscles. The Pearson correlation coefficient was applied to test the intraobserver reliability of strain imaging for assessing the biceps brachii muscle in stroke survivors. $P<.05$ was considered statistically significant. Statistical analyses were performed with SPSS version 24.0 software (IBM Corporation, Armonk, NY) and Excel version 13 software (Microsoft Corporation, Redmond, WA).

\section{Results}

From February to July 2017, we recruited 8 healthy volunteers ( 4 men and 4 women; age range, 40-56 years; mean age, 46 years) and 8 stroke survivors ( 5 men and 3 women; age range, 34-72 years; mean age, 59 years). The difference in the ages between healthy controls and stroke survivors was significant $(P=.04)$. The causes of stroke included intracranial hemorrhage $(n=4)$ and ischemic infarction $(n=4)$. One stroke survivor who did not have upper limb flexor spasticity was excluded from the data analysis. Finally, strain imaging data acquired from 7 patients with poststroke biceps brachii muscle spasticity and 8 healthy controls were analyzed. The times from the stroke to the US examination ranged from 0.6 to 24 years, with an average of 9 years. The passive range of motion in stroke survivors varied from $130^{\circ}$ to $180^{\circ}$, with an average of $164^{\circ}$. The modified Ashworth Scale was scored $2(n=4)$ and $1+(n=3)$, and the Tardieu Scale was scored $2(n=7)$ in the 7 stroke survivors. The data quality index for estimating the biceps brachii muscle and subcutaneous tissue in all participants ranged from 0.90 to 0.99 , with an average of 0.95 ( $>0.9$ is considered valid data). Strain imaging measurements are listed in Table 1. The intraobserver reliability of performing strain imaging in the biceps brachii muscle was good $(r=0.85 ; P<.01)$.

We observed a significant increase in muscle stiffness, as represented by a remarkable decrease in muscle axial strain in the spastic muscle compared with the axial strain in healthy and nonspastic muscles (all $P<.01$; Table 1 and Figure 5). The other component of spastic muscle changes is neurophysiologic, as passive muscle compliance characterized by muscle lengthening and shortening during passive elbow movement. ${ }^{28}$ Along these lines, we also found impaired biceps brachii dynamic displacement, as represented by significant decreases in longitudinal strains (lengthening, Figure $6 \mathrm{~A}$; shortening, Figure 6B), and tissue velocity (Figure 
7) in the spastic biceps brachii muscle compared with healthy and nonspastic muscles.

\section{Discussion}

We report the feasibility of strain imaging for assessing the difference in biceps brachii stiffness and passive dynamic displacement between healthy controls and individuals with chronic poststroke spasticity. Central nervous system disorders with upper motor neuron dysfunction often produce spasticity and hypertonia of the limb that is velocity dependent and dependent on the range of motion. The elbow flexors (biceps brachii muscle) are most involved and characterized by an increased resistance to passive stretching. ${ }^{5}$ In poststroke spasticity management, 2 components of muscle changes have received special attention in neurology and rehabilitation. ${ }^{29}$ One component of spastic muscle changes relates to the biomechanical properties, as passive muscle stiffness characterized by the axial deformation of an individual muscle under external compression. ${ }^{30}$ This muscle stiffness is associated with a gain of the stretch reflex and an increase in reflex stiffness and may contribute to spasticity. ${ }^{31}$ We observed a significant increase in muscle stiffness, as represented by a remarkable decrease in axial strain in the spastic biceps brachii muscle compared with the healthy and nonspastic muscles (all $P<.01$; Table 1 and Figure 5). The other component of spastic muscle changes is neurophysiologic, as passive muscle compliance characterized by muscle lengthening and shortening during passive elbow movement. ${ }^{28}$ Along these lines, we also found impaired biceps brachii dynamic displacement, as represented by significant decreases in longitudinal strains (lengthening, Figure 6A; shortening, Figure 6B) and tissue velocity (Figure 7) in the spastic biceps brachii muscle compared with healthy and nonspastic muscles.

The explanation for these findings is straightforward. When the biceps brachii muscle is spastic, it is stiffer, and muscle axial deformation and longitudinal displacement decrease (Table 1). Changes in the muscle are characterized by increased resistance to passive

Figure 5. Box-and-whisker plots show the axial strain ratios of spastic, nonspastic, and healthy biceps brachii muscles (BBM) at elbow joint angles of $90^{\circ}(\mathbf{A})$ and $0^{\circ}$ or the maximal achieved extension angle (B) in poststroke patients. The axial strain ratio in the spastic muscle is significantly lower than in healthy and nonspastic muscles (Table 1). The difference in the axial strain ratio between healthy and nonspastic muscles is also significant at elbow extension (elbow angle of $0^{\circ}$ ), but it is not at the elbow flexion angle of $90^{\circ}$.
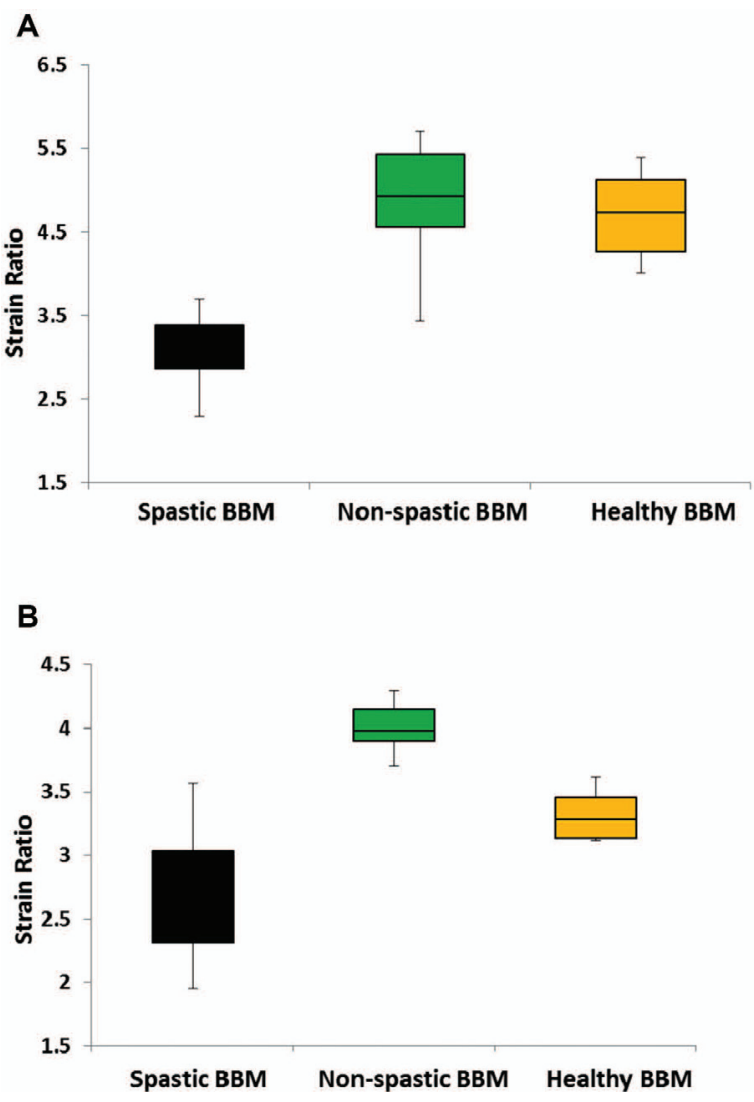

Table 1. Ultrasound Strain Imaging Parameters for the Biceps Brachii Muscle in 8 Healthy Controls and 7 Stroke Survivors

\begin{tabular}{lcccc}
\hline Parameter & Healthy & Nonspastic Biceps & Spastic Biceps & F/P \\
\hline Maximal elbow extension, & 180 & 180 & 164 & $9.10 / .001$ \\
Axial strain ratio $\left(90^{\circ}\right)$ & $4.71 \pm 0.06$ & $4.87 \pm 0.04$ & $3.13 \pm 0.46$ & $102.39 /<.0001$ \\
Axial strain ratio $\left(0^{\circ}\right)$ & $3.32 \pm 0.04$ & $4.02 \pm 0.05$ & $2.65 \pm 0.04$ & $1976 /<.0001$ \\
Longitudinal strain ratio $\left(90^{\circ}-0^{\circ}\right)$ & $4.56 \pm 0.06$ & $5.79 \pm 1.49$ & $3.12 \pm 0.68$ & $15.96 /<.0001$ \\
Longitudinal strain ratio $\left(0^{\circ}-90^{\circ}\right)$ & $4.77 \pm 0.06$ & $6.56 \pm 0.31$ & $3.25 \pm 0.74$ & $101.78 /<.0001$ \\
Tissue velocity $\left(90^{\circ}-0^{\circ}\right), \mathrm{cm} / \mathrm{s}$ & $2.14 \pm 0.48$ & $2.16 \pm 0.6$ & $1.33 \pm 0.45$ & $6.79 / .005$ \\
Tissue velocity $\left(0^{\circ}-90^{\circ}\right), \mathrm{cm} / \mathrm{s}$ & $-2.11 \pm 0.63$ & $-2.09 \pm 0.29$ & $-0.95 \pm 0.31$ & $18.34 /<.0001$ \\
\hline
\end{tabular}

$F$ value is the variance of the group means (mean squared between)/mean of the within-group variance (mean squared error). 
muscle displacement, diminished muscle velocity, and a reduced range of muscle displacement during elbow extension. These changes may be associated with the reduction of fascicle length and the elevation of wholemuscle stiffness in chronic stroke survivors. ${ }^{32}$ Although spasticity is neurologic in origin, substantial structural adaptations in the soft tissue occur, as changes in both muscle cells and the extracellular matrix contribute to the limitation of the displacement in spastic muscle. ${ }^{28}$ Therefore, the increase in stiffness in chronic spastic muscle is often associated with changes in mechanical muscle fiber properties as intramuscular adipose and connective tissues increase. ${ }^{12,29-32}$ These changes

Figure 6. Box-and-whisker plots show the longitudinal strain ratios of spastic, nonspastic, and healthy biceps brachii muscles (BBM) to reference tissue produced by elbow extension from 90 degree to 0 degree $(\mathbf{A})$ and elbow flexion from 0 degree to 90 degree (B). The longitudinal strain ratio in the spastic muscle is significantly lower than in healthy and nonspastic muscles (Table 1). The difference in the longitudinal strain ratio between healthy and nonspastic muscles at extension and flexion is also significant $(P<.05)$.

A

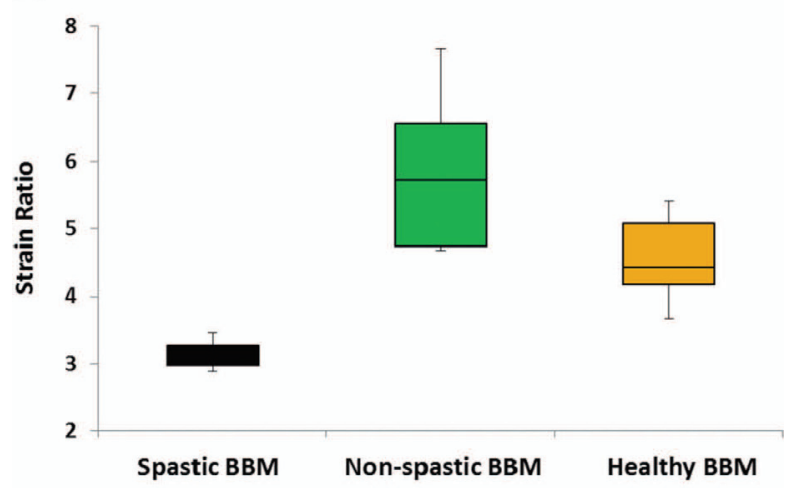

B

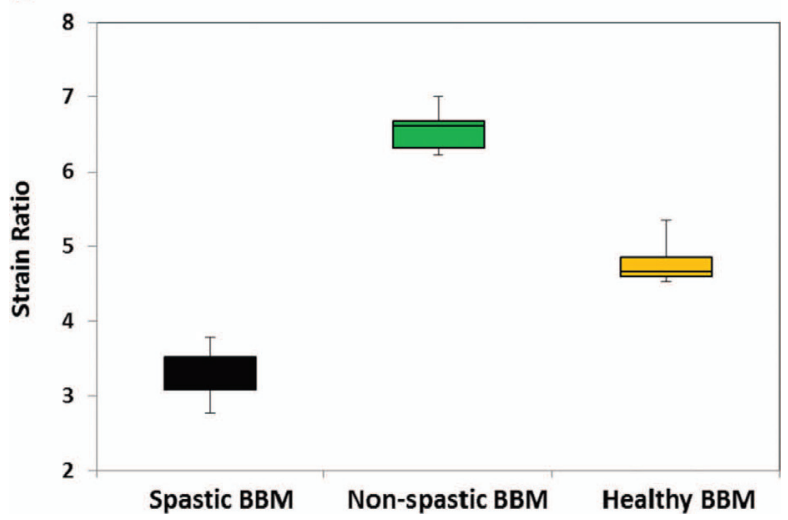

understandably lead to the decrease in biceps brachii muscle strain on strain imaging (Figure 5), as we observed in this study. In addition, biceps brachii displacement may help in evaluating the stretch reflex activity that is strongly associated with function of skeletal muscles in poststroke spasticity. There are 2 parameters that can be used to examine impaired reflex modulation of the biceps brachii muscle: longitudinal strain and tissue velocity. Longitudinal muscle strain shows slower/ decreased muscle stretching and shortened muscle length (reduced muscle fiber displacement) at the maximal stretching on a time-strain curve (Figure 3).

Figure 7. Box-and-whisker plots show peak tissue velocities in spastic, nonspastic, and healthy biceps brachii muscles (BBM) during elbow extension from $90^{\circ}$ to $0^{\circ}(\mathbf{A}$; a positive velocity value represents muscle lengthening) and during elbow flexion from $0^{\circ}$ to $90^{\circ}$ ( $\mathbf{B}$; a negative velocity value represents muscle shortening). One can clearly note that the peak velocity in the spastic muscle is significantly lower than in healthy and nonspastic muscles (Table 1) during extension and flexion. The difference in tissue velocity between healthy and nonspastic muscles is not significant $(P>.05)$.

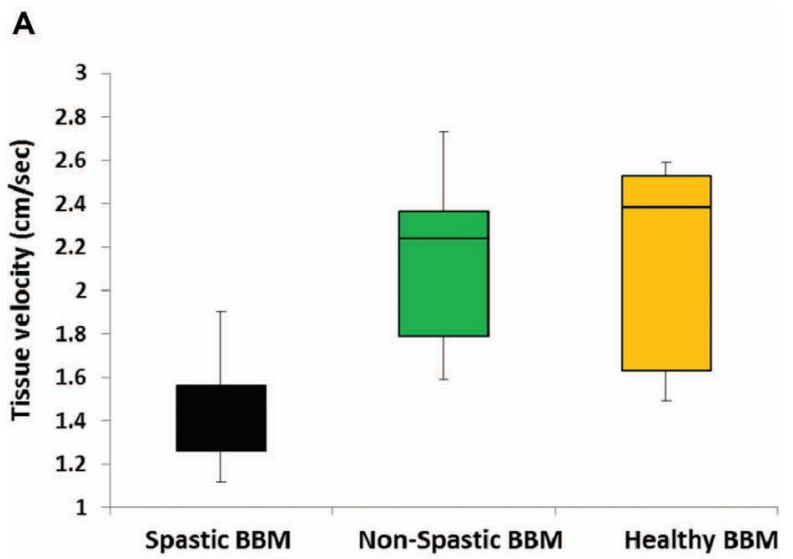

B

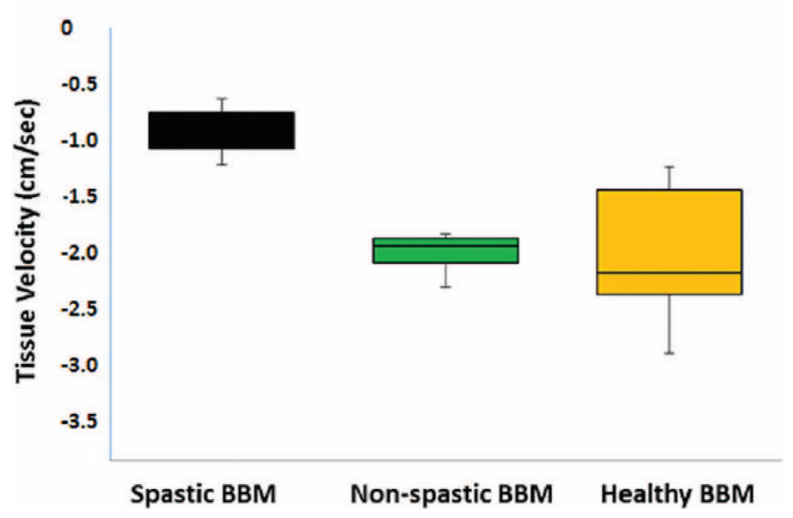


Additionally, reduced muscle stretching and increased resistance manifests on strain imaging as a decreased tissue velocity amplitude, represented on the time-velocity curve (Figure 4B). A lower tissue velocity may be associated with the response of a hypertonic elbow flexor (biceps brachii muscle) to the stretch reflex at slower speeds. ${ }^{30}$ It is important to note that all 3 strain imaging parameters (axial strain ratio, longitudinal strain ratio, and tissue velocity) are significantly decreased in spastic biceps brachii muscle and correlated with clinical assessments using the modified Ashworth Scale $(>1+$, increase in muscle tone and resistance to catch) and Tardieu Scale (2, interruption of passive movement).

Interestingly, the difference in axial strain ratios between healthy and nonspastic biceps brachii muscles at maximal elbow extension was significant $(P<.05)$. The difference in longitudinal stain ratios during muscle lengthening and shortening between healthy and nonspastic biceps brachii muscles was also significant (all $P<.01)$. These increases in axial and longitudinal strains in the nonspastic biceps brachii muscle may be associated with compensative mechanisms in stroke survivors, with spastic biceps brachii impairment, which may lead to mechanical property (axial strain) and function (longitudinal strain) changes in the contralateral muscle. ${ }^{33}$ However, the difference in biceps brachii longitudinal tissue velocities during lengthening or shortening between healthy and nonspastic biceps brachii muscles (all enrolled stroke survivors with mild biceps brachii spasticity and a modified Ashworth Scale score $<2$ ) was not significant $(P>.05$; Figure 7$)$. The difference in axial strain ratios between healthy and nonspastic biceps brachii muscles at $90^{\circ}$ of elbow flexion was not significant either $(P>.05$; Figure $5 \mathrm{~A})$. These findings may have resulted from the similarity of muscle stiffness in the relaxation, stretching resistance, and range of motion (reaching full elbow extension) in both healthy and nonspastic biceps brachii muscles.

There were limitations to this study. First, the number of enrolled patients with poststroke spasticity was small; however, significant differences were detected. Second, the difference in the ages between healthy controls and stroke survivors was significant. Since the muscle shear modulus and strength seem to be affected at ages of 60 years and older, ${ }^{34}$ and the average age in this study was 59 years, this factor could have influenced the results to some degree. Third, strain imaging was used to assess chronic poststroke spasticity, and the generalizability of these results to acute spasticity is unknown. Fourth, interobserver agreement in performing strain imaging for poststroke spasticity was not formally tested, although good intraobserver reproducibility of strain imaging was observed. Fifth, electromyographic tracing was not used to monitor muscle activity while assessing real-time muscle movement in the study. In addition, the force used to produce muscle deformation and displacement may have varied in some degree, even though we used a $1.0-\mathrm{kg}$ sandbag as a standard external compression force to produce axial biceps brachii deformation, and we normalized biceps brachii axial strain and longitudinal strain by using axial and longitudinal reference subcutaneous strains in all participants. Finally, biceps brachii movement is 3-dimensional, whereas we estimated 1-dimensional muscle strain. Therefore, further studies with greater numbers of participants in larger age groups with acute and chronic poststroke spasticity and the possibility of using 3-dimensional strain techniques will likely provide additional information.

In conclusion, our results suggest that US strain imaging is a useful imaging tool for determining increased stiffness and decreased dynamic displacement in spastic biceps brachii muscles by assessing the axial strain, longitudinal strain, and tissue velocity of the muscle. These noninvasive strain imaging markers may have potential in improving the point-of-care management of chronic poststroke spasticity.

\section{References}

1. Thibaut A, Chatelle C, Ziegler E, Bruno MA, Laureys S, Gosseries O. Spasticity after stroke: physiology, assessment and treatment. Brain Inj 2013; 27:1093-1105.

2. Wissel J, Schelosky LD, Scott J, Christe W, Faiss JH, Mueller J. Early development of spasticity following stroke: a prospective, observational trial. J Neurol 2010; 257:1067-1072.

3. Urban PP, Wolf T, Uebele M, et al. Occurrence and clinical predictors of spasticity after ischemic stroke. Stroke 2010; 41:2016-2020.

4. Doan QV, Brashear A, Gillard PJ, et al. Relationship between disability and health-related quality of life and caregiver burden in patients with upper limb poststroke spasticity. PM R 2012; 4:4-10.

5. Starky A, Sangani SG, McGuire JR, Logan B, Schmit BD. Reliability of biomechanical spasticity measurements at the elbow of people poststroke. Arch Phys Med Rehabil 2005; 86:1648-1654.

6. Bilston LE, Tan K. Measurement of passive skeletal muscle mechanical properties in vivo: recent progress, clinical application, and remaining challenges. Ann Biomed Eng 2015; 43:261-273. 
7. Sommerfeld DK, Gripenstedt U, Welmer AK. Spasticity after stroke: an overview of prevalence, test instruments, and treatments. Am J Phys Med Rehabil 2012; 91:814-820.

8. Pisano F, Miscio G, Conte C, Pianca D, Candeloro E, Colombo R. Quantitative measures of spasticity in post-stroke patients. Clin Neurophysiol 2000; 111:1015-1022.

9. Ettema GJC, Huijing PA. Skeletal muscle stiffness in static and dynamic contractions. J Biomech 1994; 27:1361-1368.

10. Lee SS, Spear S, Rymer WZ. Quantifying changes in material properties of stroke-impaired muscle. Clin Biomech 2015; 30:269-275.

11. Wu CH, Ho YC, Hsiao MY, Chen WS, Wang TG. Evaluation of post-stroke spasticity muscle stiffness using shear wave ultrasound elastography. Ultrasound Med Biol 2017; 43:1105-1111.

12. Chen EJ, Jenkins WK, O'Brien WD Jr. Performance of ultrasound speckle tracking in various tissues. J Acoust Soc Am 1995; 98:1273-1278.

13. Chino K, Akagi R, Dohi M, Takahashi H. Measurement of muscle architecture concurrently with muscle hardness using ultrasound strain elastography. Acta Radiol 2014; 55:833-839.

14. Niitsu M, Michizaki A, Endo A, Takei H, Yanagisawa O. Muscle hardness measurement by using ultrasound elastography: a feasibility study. Acta Radiol 2011; 52:99-105.

15. Lubinski MA, Emelianov SY, O’Donnell M. Speckle tracking methods for ultrasonic elasticity imaging using short-time correlation. IEEE Trans Ultrason Ferroelectr Freq Control 1999; 46:82-96.

16. Varghese T. Quasi-static ultrasound elastography. Ultrasound Clin 2009; 4:323-338.

17. O'Donnell M, Skovoroda AR, Shapo BM, Emelianov SY. Internal displacement and strain imaging using ultrasound speckle tracking. IEEE Trans Ultrason Ferroelectr Freq Control 1994; 41:314-325.

18. Sikder S, Wei Q, Cortes N. Dynamic ultrasound imaging application to quantify musculoskeletal function. Exerc Sport Sci Rev 2014; 42: 125-135.

19. Gao J, He W, Du LJ, et al. Ultrasound strain elastography in assessment of resting biceps brachii muscle stiffness in patients with Parkinson's disease: a primary observation. Clin Imaging 2016; 40:440-444.

20. Gao J, Li PC, Chen J, et al. Ultrasound strain imaging in assessment of biceps muscle stiffness and dynamic motion in healthy adults. Ultrasound Med Biol 2017; 43:1729-1736.

21. Kaluzynski K, Chen XC, Emelianov SY, Skovoroda AR, O’Donnell M. Strain rate imaging using two-dimensional speckle tracking. IEEE Trans Ultrason Ferroelectr Freq Control 2001; 48:1111-1123.
22. Patel P, Biswas R, Park DW, et al. Characterization of vascular strain during in-vitro angioplasty with high-resolution ultrasound speckle tracking. Theor Biol Med Model 2010; 7:36.

23. D'hooge J, Heimdal A, Jamal F, et al. Regional strain and strain rate measurements by cardiac ultrasound: principle, implementation, and limitations. Eur J Echocardiogr 2000; 1:154-170.

24. Grönlund C, Claesson K, Holtermann A. Imaging two-dimensional mechanical wave of skeletal muscle contraction. Ultrasound Med Biol 2013; 39:360-369.

25. Peolsson M, Brodin LA, Peolsson A. Tissue motion pattern of ventral neck muscles investigated by tissue velocity ultrasonography imaging. Eur J Appl Physiol 2010; 109:899-908.

26. Lindberg F, Mårtensson M, Grönlund C, Brodin LA. Evaluation of ultrasound tissue velocity imaging: a phantom study of velocity estimation in skeletal muscle low-level contraction. BMC Med Imaging 2013; 13:16.

27. Pandyan AD, Price CI, Barnes MP, Johnson GR. A biomechanical investigation into the validity of the modified Ashworth Scale as a measure of elbow spasticity. Clin Rehabil 2003; 17:290-293.

28. Haugh AB, Pandyan AD, Johnson GR. A systematic review of the Tardieu Scale for the measurement of spasticity. Disabil Rehabil 2006; 28:899-907.

29. Lieber RL, Ward SR. Cellular mechanisms of tissue fibrosis, 4: structural and functional consequences of skeletal muscle fibrosis. Am J Physiol Cell Physiol 2013; 305:C241-C252.

30. Mayer N, Esquenazi A, Childers M. Common patterns of clinical motor dysfunction. Muscle Nerve 1997; 6:S21-S35.

31. Thilmann AF, Fellows SJ, Garms E. The mechanism of spastic muscle hypertonus: variation in reflex gain over the time course of spasticity. Brain 1991; 114:233-244.

32. Gao F, Grant TH, Roth EJ, et al. Changes in passive mechanical properties of the gastrocnemius muscle at the muscle fascicle and joint levels in stroke survivors. Arch Phys Med Rehabil 2009; 90:819-826.

33. Lorentzen J, Grey MJ, Crone C, Mazevet D, Biering-Sørensen F, Nielsen JB. Distinguishing active from passive components of ankle plantar flexor stiffness in stroke, spinal cord injury and multiple sclerosis. Clin Neurophysiol 2010; 121:1939-1951.

34. Eby SF, Cloud BA, Brandenburg JE, et al. Shear wave elastography of passive skeletal muscle stiffness: influences of sex and age throughout adulthood. Clin Biomech 2015; 30:22-27. 